\title{
The Design of Integrated Wind Turbine Pitch Controller
}

\section{Chen Xi ${ }^{1, \text { a }}$, Qiu Xiaowei ${ }^{1, b}$, Li Peng ${ }^{1, c}$, Zhang Jun ${ }^{1, d}$, Zhang Xing ${ }^{2, e}$}

\author{
${ }^{1}$ Beijing Huadian Tianren Electric Power Control Technology CO.,LTD, No.1 Building, No.16 West
} 4th Ring Road, HaiDian District, Beijing,China,100039,

${ }^{2}$ GuoDian United Power Technology CO. LTD, No.1 Building, No.16 West 4th Ring Road, HaiDian District, Beijing,China, 100039,

achenxi1@gdtianren.com, 'o qiuxiaowei@gdtianren.com, 9li_peng@gdtianren.com, 'zhangjun@gdtianren.com, èzhangxing@gdupc.cn

\section{Keywords: Integrated. Wind Turbine. Pitch Controller. PLC. Servo Drive}

Abstract. In traditional structure, wind turbine pitch control system generally consists of two modules, the logic control unit responsible for the control logic and the servo drive unit responsible for the motor driven. In order to adapt to the integration of the system architecture and simplify complex layout and cabling, this study designs a full function controller integrated with double-PCB structure programmable logic controller (PLC) and a low-voltage asynchronous servo drive, to develop a new structure which puts the PLC and servo drive in one metal chassis. In addition, a double-fault-diagnosis logic was designed to improve security. The practical industrial application shows this design improves the safety and stability performance of wind turbine pitch control system, while obtaining good economic benefits.

\section{Introduction}

With the growing rating of the wind energy generation system, as well as wind turbine runtime environment changes from inland to offshore, the reliability of the wind turbine pitch control system are more demanding than ever before [1]. The fault problems of wind turbines have become increasingly prominent, and become a research focus of the various scientific research institutions at home and abroad [2,3]. Fault statistics can be seen from domestic and foreign wind turbines, in which the pitch system is one of the high incidences of fault mechanical components [4]. In general, the traditional pitch control system includes complex layout, cabling, and tedious production processes [5]. As we know cables and connecting elements are easy to age and have vulnerability to environmental factors such as temperature, moisture and electromagnetic interference.

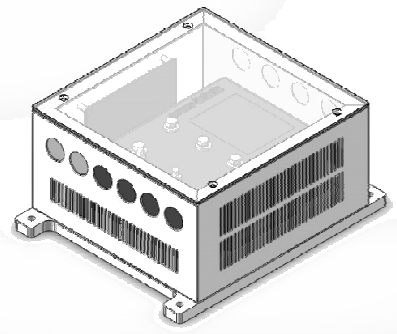

Figure 1.Structure schematic of integrated controller

In order to solve these problems, we designed a completely self-developed integral wind turbine pitch controller, which integrated a full function PLC and a low-voltage asynchronous servo drive within one metal chassis as showed in Fig.1. The PLC is responsible for system control, system security protection and fault detection, etc. Servo drive executes pulse commands from the PLC, which always are classified into DC drives, AC asynchronous drive and $\mathrm{AC}$ synchronous drive by driving mode. With the development of AC motor control technology, DC pitch control system was replaced by asynchronous pitch control system and permanent magnet pitch control system because of its brush $[6,7]$. We chose synchronous drive in our design. 


\section{The Integrated Structure Design of Pitch Controller}

In this section we present how the controller is connected in pitch control system, and the integrated structure of this controller. Moreover, main technical index is listed finally contains performance we achieved.

The integrated controller concludes a PLC and servo drive in one chassis. As is shown in Fig.2, we designed double CAN bus interfaces for communication, extension and redundancy between master controller and pitch control system. Multi-channel high speed sync IO interfaces were designed for acquisition and output of switch variables, acquisition of SSI signal from encoder and acquisition of thermal resistance signal. Users are allowed to browse pitch control system real-time data from HMI on upper computer through connecting Ethernet interfaces equipped on integrated controller.

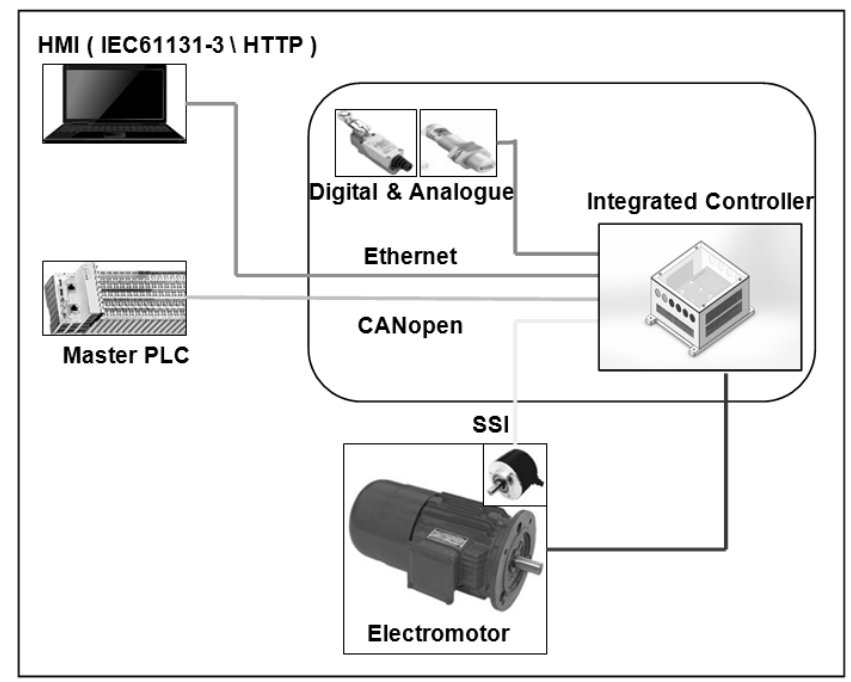

Figure 2. Hardware connection of integrated controller

A new full function double-PCB structure PLC was designed for the controller, including CPU-PCB and IO-PCB as shown in Fig.3. On the CPU-PCB, the low-power EP9302 chip which based on ARM9 architecture from CirrusLogic was took as the system main processor, with $200 \mathrm{MHz}$ frequency, $32 \mathrm{MB}$ SDRAM and $16 \mathrm{MB}$ NORFLASH. Ethernet interfaces and CANopen interfaces are equipped on the side of CPU-PCB. For processing large data sets faster, we selected MSP430 from TI as slave CPU on IO-PCB, with multi-channel general-purpose I/O (GPIO) and high precision ADC. Meanwhile tow 26-bit IO terminals S2L 3.5/90F from Weidmuller routing parallel to PCBs were allocated. Then these two PCBs work cooperatively to complete the control task with much less space but more stable in structure than traditional modular structure plc.
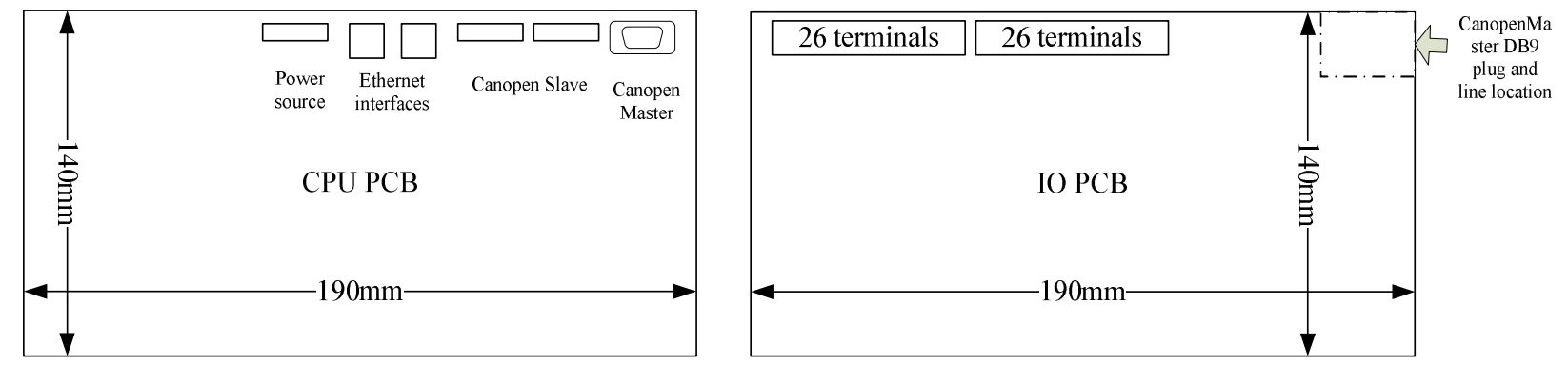

Figure 3. Schematic diagram of PCB of PLC

The structure inside integrated controller can be seen in Fig. 4, in which the servo drive is fixed perpendicular to double-PCB PLC in the chassis. This structure has natural advantage in resistance to dust and vibration, which is extremely important in wind turbine pitch control system. In addition, 
differing from independent plc and servo drive modules, this integrated design overcomes the effect from intermediate line to the performance of the control efficiency and safety.

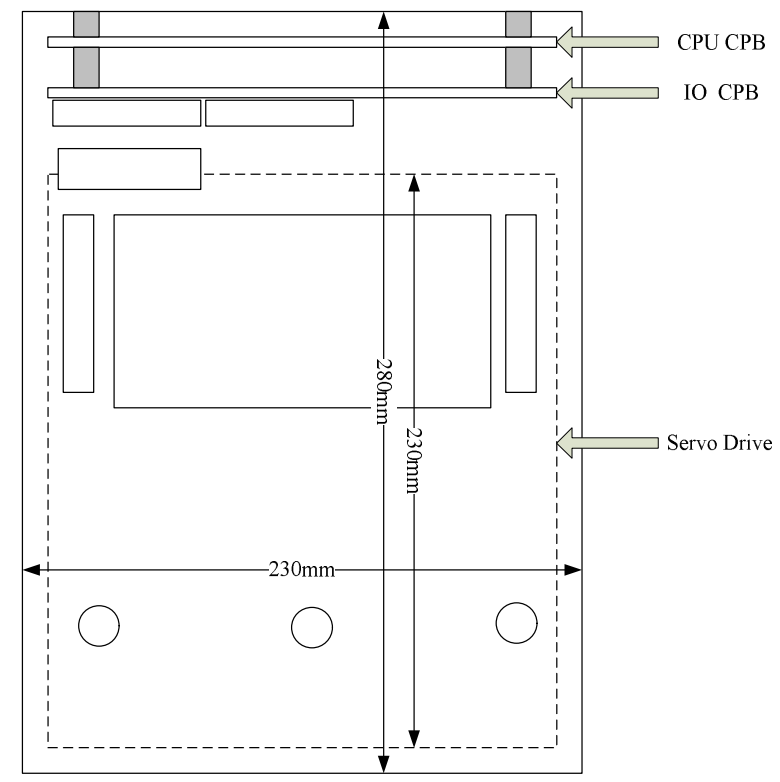

Figure 4. Structure graphing of integrated controller

With the integrated structure, our controller ensures conformance to the technical index showed in table 1, and has the following features:

a) The integration of the appearance and structure;

b) Unified IO interface;

c) The low voltage induction servo drive function;

d) Programmable and configurable PLC function (support full IEC61131-3 Standard);

e) The fieldbus communication (Canopen, Profibus);

f) Extensible high-speed synchronous IO.

Table 1. The main technical index of integrated controller

\begin{tabular}{|c|c|}
\hline ITEM & PARAMETER \\
\hline \multirow{2}{*}{ Performance of PLC } & IO Bus Access Time $<4 \mathrm{~ms}$ \\
\hline & Minimum Run Cycle: $10 \mathrm{~ms}$ \\
\hline \multirow{2}{*}{ Performance of fieldbus } & Canbus Baud Rate $>500 \mathrm{KHz}$ \\
\hline & Profibus Baud Rate $>=1500 \mathrm{KHz}$ \\
\hline \multirow{4}{*}{ IO type supported } & 16 Channels Active Digital Input(DI) \\
\hline & 16 Channels Active Digital Output(DO) \\
\hline & 4 Channels Resistance Temperature Detector(RTD) \\
\hline & SSI Analog Input(SSI) \\
\hline \multirow{3}{*}{ Performance of Servo Drive } & Operating Voltage $24 \mathrm{~V}, 36 \mathrm{~V}, 48 \mathrm{~V}, 72 / 80 \mathrm{~V}$ \\
\hline & $\begin{array}{l}\text { Maximum Current( }(3 \text { minutes }) 500 \mathrm{~A}(24 \mathrm{~V}), 450 \mathrm{~A}(36 \mathrm{~V}) \text {, } \\
450 \mathrm{~A}(48 \mathrm{~V}), 275 \mathrm{~A}(72 / 80 \mathrm{~V})\end{array}$ \\
\hline & Operating Frequency $8 \mathrm{kHz}$ \\
\hline
\end{tabular}

\section{The Software Design of Pitch Controller}

Software on master CPU and slave CPU were programed independently, achieving separate faults diagnosis processing logic and double emergency protecting function. The master CPU is responsible for the communication with master PLC, internal communication and management of variable frequency drive, fault detection and process, the servo position loop operation and human-machine interface function (HMI). While the slave CPU is processor core of IO processing, communicating with master CPU by SPI bus. 
PLC runtime was designed on slave CPU for system scheduler, as well as interface driven was designed such as SPI driver and can bus driver. A CANopen protocol stack is transplanted on CPU which is necessary for communicated with master PLC.

SPI driver. The main functions of SPI driver list as follow:

void ssp_init(void)

SPI IO initialization, including IO configuration, interrupt setting, baud rate and clock setting.

static void ssptx_interrupt(int irq, void *dev_id, struct pt_regs *regs)

SPI message send interrupt.

static void ssprx_interrupt(int irq, void $* d e v \_i d$, struct pt_regs *regs)

SPI message receive interrupt.

int ssp_ioctl(struct inode *inode, struct file *filp, unsigned int $\mathrm{cmd}$, unsigned long arg)

User interface functions of SPI, in which the 'cmd' parameter represents the command what user want to execute. Specifically, 'cmd' defined as macro as follow:
\#define IOCTL_CMD_SSPTX
1
\#define IOCTL_CMD_GRST 2
\#define IOCTL_CMD_GETVERSION 19
//SPI IO send
//Reset All IO Modular
//Read driver version number

Transplant of CANopen protocol stack. To match to the CANopen protocol stack, we reprogrammed driver and scheduler program related to the hardware.

static void sjal000_chip_init(void)

SJA1000 initialization.

static int sja1000_irq_handler(void)

SJA send \& receive interrupt.

static void dnamtimer2_interrupt(int irq, void *dev_id, struct pt_regs *regs)

interrupt of Canfestival Finite State Machines timer.

int sja1000_ioctl(struct inode *inode, struct file *filp, unsigned int cmd, unsigned long arg)

User interface functions of CAN, in which the 'cmd' defined as macro as follow:

\#define CANOPENSLAVE_DR_WAITSYNC O // timerfor SYNC

\#define CANOPENSLAVE_DR_READ 1 //PDO read

\#define CANOPENSLAVE_DR_WRITE 2 I/PDO write

\#define CANOPENSLAVE_DR_GETTIMESTAMP 3 //get time stamp for fault diagnosis

\#define CANOPENSLAVE_DR_INIT 4 //Initialization of protocol stack

Fault diagnosis logic. Aim to improve safety, the slave CPU is designed an independent faults diagnosis processing logic separated from master CPU. As any traditional plc, the master CPU monitors real-time data. The whole system could face the wind automatically with blade angles turn to $85^{\circ}$ when controller finds the data may be not correct or receive halt instruction from master PLC. The turbine will enter emergency fault state which we called fault mode 1 . In this mode the controller can be self-reset.

In addition, to achieve double fault diagnosis, the slave CPU will boot turbine into fault mode 2 when judging master CPU in abnormal state from heartbeat, which make the wind turbine into a state of emergency stop with blade angles turn to $95^{\circ}$. This mode cannot be self-reset for security purpose, as the master CPU may be damaged. Until the fault is excluded and manual reset again the wind turbine will restore normal operation. Double processing logic is shown in Fig.5. 


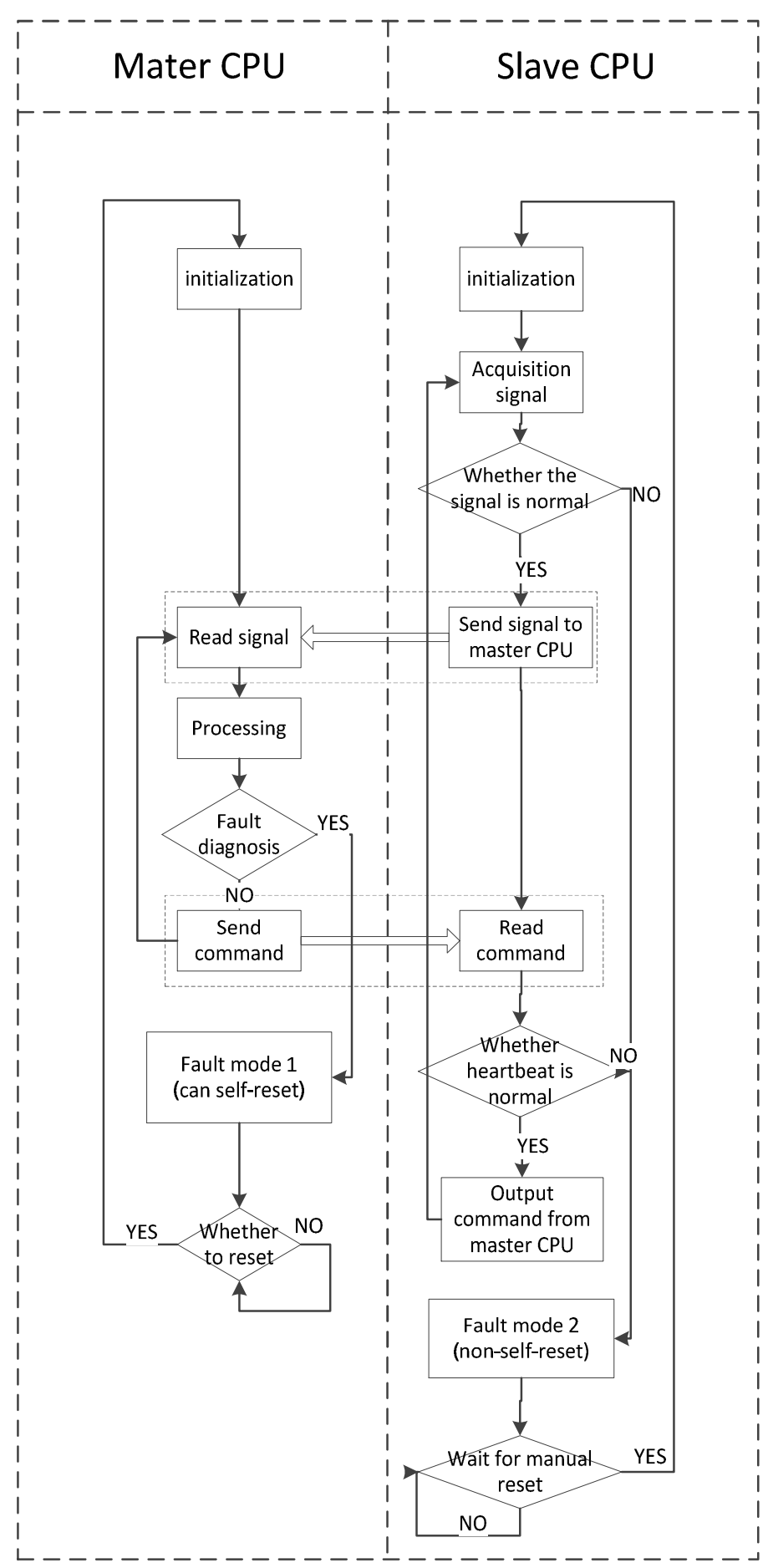

Figure 5. Double processing logic for faults diagnosis

\section{Conclusions}

The design of integrated wind turbine pitch controller concludes structure design, hardware design and software design. This completely self-developed controller has an integrated structure contributing to get a natural advantage in resistance to dust and vibration, as well as overcome the effect from intermediate line to the performance of the control efficiency. We designed double CPU contributing to higher data processing capacity and coded double-fault-diagnosis logic contributing to ensure security of wind turbine system. Now this controller has been successfully applied in a demonstration project, effectively decreasing fault rate and reduced the system cost. 


\section{References}

[1] N.Horiuchi, T.Kawahito. Torque and power limitations of variable speed wind turbines using pitch control and generator power control[C].Power Engineering Society Summer Meeting 2001.IEEE, 2001(1):638-643.

[2] Tavner P J, Xiang J, Spinato F.Reliability analysis for wind turbines, Journal of Wind Energy Vol. 10(2006) No.1, 1-18.

[3] Zhang Zhen, Guan Shuqiang: An analysis of wind turbine fault statistics, Wind Energy, Vol.8(2013), 68-71.

[4] Liu Junqiao, Marwan Rosyadi, Rion Takahashi,etc. A new pitch control system of wind turbine to damp power system frequency fluctuations[C]. Renewable Energy Research and Applications (ICRERA). IEEE, 2012.

[5] K.Vijaya, Lakshmi, P.Srinivas. Fuzzy Adaptive PID Control of Pitch System in Variable Speed Wind Turbines[C]. 2014 International Conference on Issues and Challenges in Intelligent Computing Techniques (ICICT).IEEE, 2014:52-57.

[6] Phongsak, Phakamach. Control of a DC Servomotor Using Fuzzy Logic Sliding Mode Model Following Controller [J].World Academy of Science, Engineering and Technology, 2009, 5(1):504-509.

[7] Lin B R, Yang T Y. Three-phase AC/DC converter with high power factor[J]. Electric Power Applications. 2005. 152(3): 757-764. 